\title{
Advanced analysis of backsheet failures from 26 power plants
}

\author{
Jochen Markert*, Sandra Kotterer, Djamel Eddine Mansour, Daniel Philipp, and Paul Gebhardt \\ Fraunhofer Institute for Solar Energy Systems, Heidenhofstr. 2, 79110 Freiburg, Germany
}

Received: 30 June 2021 / Received in final form: 30 September 2021 / Accepted: 21 October 2021

\begin{abstract}
Backsheet degradation is a known reliability issue affecting field-exposed photovoltaic (PV) modules power plants. In this work, we present lessons learned during the last three years, examining modules from 26 power plants in the TestLab PV Modules at Fraunhofer ISE. The basis is a description of the currently observed backsheets and associated degradation features as for example backsheet chalking, cracks in different layers and chemical changes in composition. Furthermore, we lay out analytical methods for initial and more detailed analysis of the failures and module materials. For example, a method designated as "flashlight test" has been found to provide a quick and straightforward method to identify damaged polypropylene (PP) layers within backsheets. Furthermore, scanning acoustic microscopy (SAM) and a comparison of different variants of FTIR spectroscopy are presented.
\end{abstract}

Keywords: PV modules / backsheet degradation / scanning acoustic microscopy / insulation failure analysis

\section{Introduction}

Backsheet degradation phenomena in field-exposed PV modules have been reported for several years already and are still an issue that affects many power plants currently in operation [1-3]. This issue can cause insulation losses which in severe cases coincides with inverter outages. Along with this, safety concerns are justified, and safe operation of the plant might no longer be provided. Furthermore, a degraded backsheet can initiate secondary moisturedependent degradation processes inside the module, e.g., corrosion, which additionally impair module performance in the long term [4]. An improved understanding of backsheet degradation mechanisms [5] is essential to determine cost-effective detection and mitigation procedures [3]. This knowledge can be utilized to develop alternative materials to avoid similar issues in the future. Using the herein presented analytical data from root cause analysis projects from 26 affected power plants with a total of 279 open rack mounted PV modules analyzed in our lab, our first goal is to share our general experience and practical approach on how to handle a case of possible backsheet degradation. The investigated modules had been in the field for varying periods, but for a minimum of 4 years. As shown by Eder et al. on the EUPVSEC 2021 however, the time of exposure alone does not solely determine for the degree of degradation, but the climatic conditions on the respective location have a significant

\footnotetext{
* e-mail: jochen.markert@ise.fraunhofer.de
}

influence as well. Furthermore, we lay out typical consequences of backsheet degradation to demonstrate practical relevance of the issue. In comparison with other studies based on large-scale field inspections, the presented work focuses explicitly on results obtained in the lab. This allows a more detailed analysis and evaluation of the modules based on reproducible measurements at controlled conditions (e.g., during insulation measurements) and sophisticated analysis methods.

In a second part, this work shows our latest progresses to qualify and improve characterization methods for the analysis of solar backsheets based on three examples. These approaches can help (a) to visualize and localize degradation sites inside the material stack and thereby constitute the basis for an evaluation of the modules and (b) understand the physico-chemical mechanisms during degradation.

The identification of modules affected by backsheet degradation is a critical step, because often not all modules of a given power plant contain the same backsheet materials, even if they are of the same module type. To this end, we evaluate an easy and low-tech approach to detect inner backsheet failures on site or in the lab, using only a flashlight. Another approach is to identify the polymers present in the layer structure with spectroscopic methods. To this end, Eder et al. have shown the possibility to identify the layer stacks on-site with spectroscopic methods (Raman and NIR) [6]. However, on-site analysis of the material is typically limited to known layer stacks, that can be classified and might be not suitable to identify. The herein introduced and validated approach of FTIR 


\begin{tabular}{|c|c|}
\hline & $\begin{array}{c}\text { Backsheet layer } \\
\text { compositions found }\end{array}$ \\
\hline 1 & $\begin{array}{c}3 \times \text { polyamide layers } \\
(A A A)\end{array}$ \\
\hline 2 & F-C/PET/PP \\
\hline 3 & PET/PET/PET \\
\hline 4 & PVDF/PET/EVA \\
\hline 5 & PVDF/PET/PET \\
\hline 6 & PVDF/PET/PVDF (TPT) \\
\hline 7 & PVDF/PET/E-Layer (TPE) \\
\hline 8 & PVDF/PET/PE \\
\hline 9 & PA/PET/PA (APA) \\
\hline 10 & PE/PET \\
\hline 11 & PET/PET/EVA \\
\hline
\end{tabular}

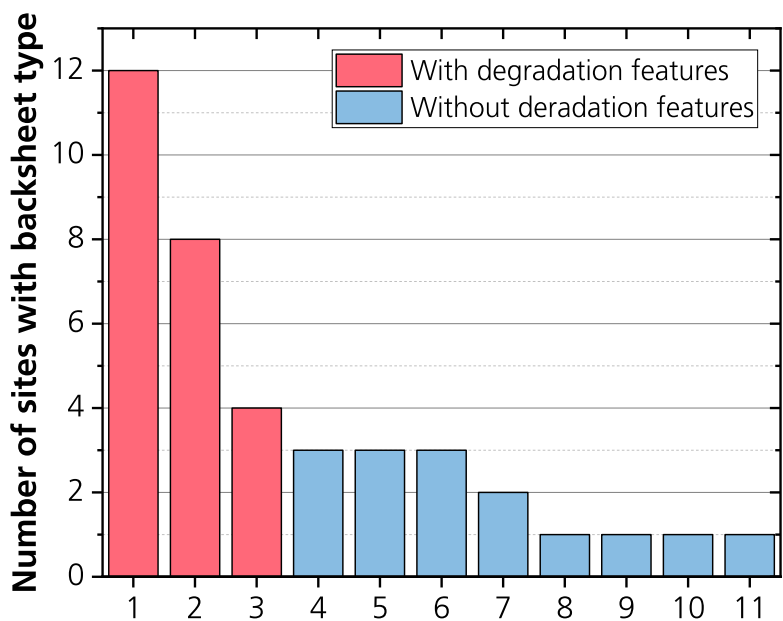

Fig. 1. Left: List of layer structures found in the investigated backsheets; Right: Graph showing the number of sites on which each respective backsheet type was found.

microscopy combines optical microscopy and FTIR spectroscopy and thereby allows to accurately select backsheet layers for spectroscopic analysis. Additionally, acoustic imaging, including micrographs and acoustic cross-section via scanning acoustic microscopy (SAM) are used to locate the backsheet cracking in specific interlayers in two different field-exposed PV modules.

\section{Methodology}

For Fourier Transform Infrared (FTIR) spectroscopy measurements, the Universal attenuated total reflection (ATR) mode Sampling Accessory from PerkinElmer was used. The surface backsheet samples were cut from the PV modules and pressed on a $\mathrm{Zn} /$ Se crystal with diamond tip. These spectra were measured in the interval $4000 \mathrm{~cm}^{-1}$ to $450 \mathrm{~cm}^{-1}$, averaging $16 \mathrm{scans}$ with a resolution of $4 \mathrm{~cm}^{-1}$. For the preparation of cross-section samples, pieces of backsheets were carefully extracted from the rear side with a utility knife in-between the cells and embedded in epoxy resin, ground and polished to allow subsequent analysis of the backsheet cross-sections with an Olympus reflectedlight microscope.

For the FTIR microscope measurements, a Bruker Hyperion 2000 was used in transmission mode. A connected Schwarzschild objective with 15x magnification was used for both imaging and spectroscopic measurements [7]. The cross-section samples were placed under the objective on a motorized stage that can be controlled on the $x-y$ plane. Before measurement, the microscope was focused onto the sample and the measurement range was limited with an aperture.

The scanning acoustic microscope used was a SAM 500 $\mathrm{HD}_{2}$, PVA TePla GmbH. The full-size PV modules were placed in a coupling fluid (in this case water) that serves as the carrier medium for the acoustic waves. The measurements were carried out in pulse-echo mode, at a normal incidence angle at the $15 \mathrm{MHz}$ acoustic frequency and at water temperatures of $>20^{\circ} \mathrm{C}$.

\section{Results and discussion}

Data of 26 projects concerning degraded backsheets in affected power plants have been collected and evaluated at the TestLab PV Modules of Fraunhofer ISE. Experiences from these investigations revealed that in such power plants, PV modules exhibit a large variety of module types from different manufacturers. The backsheets found in these modules possessed different layer structures, as listed in Figure 1. The corresponding abbreviations are provided within the following Table 1. In 11 projects, different backsheets could be identified in modules of the same type (i.e., product name). This suggests that the use of diverse backsheets is common practice in module production. This aspect complicates the selection procedure for module analysis or when identifying degraded modules from one type, e.g., for replacement without support from the module manufacturer. In some occasions, connections between the serial number and the bill of materials (BOM) could be made, e.g., when information on the production site or date is incorporated in the serial number. In other cases, visual clues could be obtained from different components (J-box, frame) or varying degree of visible degradation.

Polyamide backsheets were the most common types (reported from 12 sites) which were found during analysis and is certainly a known critical material [8]. Other critical material stacks were $\mathrm{F}-\mathrm{C} / \mathrm{PET} / \mathrm{PP}$ ( 8 sites) and $\mathrm{PET}$ based structures (e.g., PET/PET/PET) (4 sites). Backsheets $1-3$ from the table in Figure 1 (left) revealed to be the most relevant backsheets in terms of degradation and associated failure modes are shown and quantified in Figure 2. However, it is not clear to what degree these findings are representative for all backsheet products that contain these material combinations, because quality can range between different manufacturers and suppliers. It is merely an indication that at least some backsheets containing these materials showed critical degradation after field-exposure. Backsheet structures 4-11 listed in Figure 1 (left) were inconspicuous in terms of degradation 
Table 1. Abbreviations of materials used throughout this article.

\begin{tabular}{ll}
\hline Abbreviation & Material \\
\hline EVA & Ethylene vinyl acetate \\
F-C & Fluoropolymer coating \\
PA & Polyamide \\
PE & Polyethylene \\
PET & Polyethylene terephthalate \\
PP & Polypropylene \\
PVDF & Polyvinylidene fluoride \\
\hline
\end{tabular}

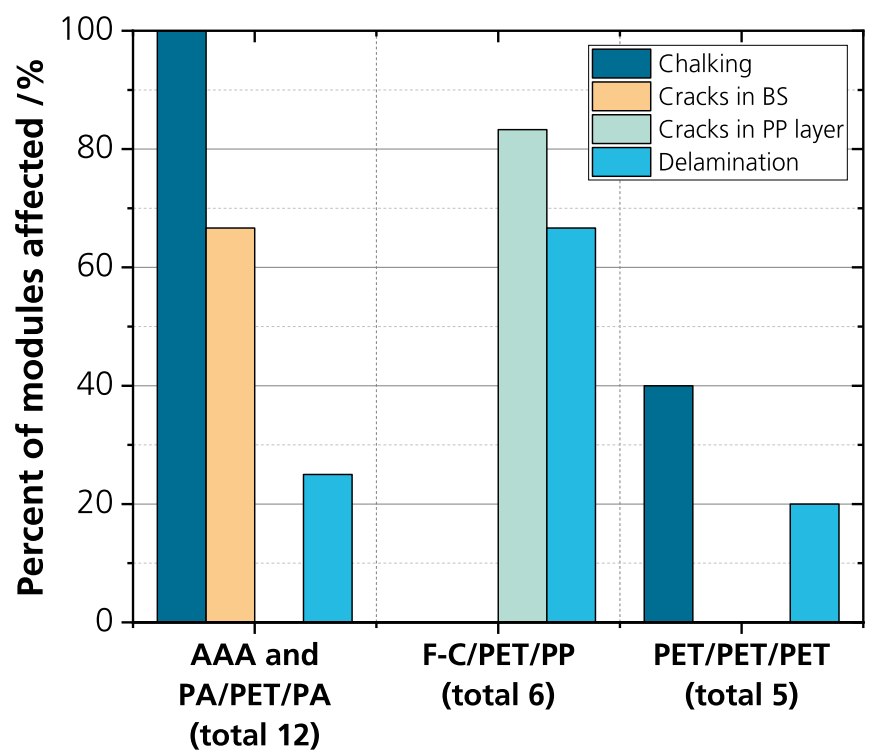

Fig. 2. Distribution of the failures in the most relevant degraded backsheet structures after several years of operation.

and did not show visual evidence for an increased degradation level in the investigated cases. However, these materials were often found in the same module types that showed damaged backsheets as mentioned above.

All modules with polyamide backsheets (AAA and PA/ $\mathrm{PET} / \mathrm{PA}$ ) as well as $40 \%$ of the PET based backsheets deposited a white powder (Fig. 3a), known as chalking [9], on the rear surface after field exposure. $67 \%$ of the investigated backsheets with polyamide developed cracks Figure 3b, typically in the cell gaps, suggesting a degradation mechanism initiated by front side UV radiation [2], or along the cell connectors, indicating the appearance of thermo-mechanical stresses in these regions. The PET based foils, on the other hand, showed no visual defects besides chalking and insulation issues have reported from one module only. This suggests that there might be a connection between chalking and the formation of backsheet cracks in polyamide layer structures. Furthermore, it has been demonstrated that, moisture inside the polymer plays a synergistic role with UV in the formation of backsheet cracking $[10,11]$. Backsheet chalking in AAA layer structures can therefore be evaluated as an early warning sign that indicates an elevated probability for the presence of a critical material and possible crack development in the future.

Typical consequences of cracks within the backsheet structure were significantly reduced insulation properties of the modules leading to an elevated safety risk and increased the chances of inverter outages, as it had been reported from six of the power plants. During the investigation in our lab, 46 modules from nine different power plants, containing AAA, PET/PET/PET and F-C/ $\mathrm{PET} / \mathrm{PP}$ designs failed the wet leakage current (WL) test according to IEC 61215-2:2016 (MQT 15) [12]. In 39 of the 46 modules an AAA backsheet could be identified. The relatively low number of only one module with a PET/ $\mathrm{PET} / \mathrm{PET}$ backsheet and the remaining six with a F-C/ $\mathrm{PET} / \mathrm{PP}$ structure suggest, that polyamide based backsheets are especially prone to insulation issues that develop during field operation, although the fraction of these materials in all power plants is unknown.

Five additional modules that passed the first WL test were subjected to an extended wetting time in a water bath for $14 \mathrm{~h}, 15 \mathrm{~h}$ and $65 \mathrm{~h}$, respectively, before the WL test was applied for a second time. This was done in order to reproduce low insulation resistance values that had been observed in the field and had led to inverter outages. All five modules used the same backsheet foil: F-C/PET/PP. The measurements showed a strong decrease of the insulation resistance values in the modules after the extended wetting time (Fig. 4), which coincides well with the observed inverter outages. A control experiment on field-aged modules without detectable backsheet degradation, in contrast, showed no significant change in insulation properties upon longer wetting time. This suggests that an increased wetting time beyond the test conditions according to IEC 61215-2:2016 (MQT 15) can be required to detect low insulation properties that lead to inverter outages.

Hidden damages in the inner PP layer were found in F-C/PET/PP structures, despite a regular outer appearance of the backsheet. Cross-sectional images (Fig. 6, left) revealed severe damage in the cell gaps within the backsheet in more than $80 \%$ of the investigated modules with this backsheet type. The location between the cell gaps indicates one more time a degradation mechanism due to UV radiation. Illumination of the modules from the front side, sometimes referred to as "flashlight test" was found to be a straightforward and cheap method to identify affected modules. This can be done with a flashlight, shining through the backsheet in the cell gaps. On the rear side, the shadows of the tiny cracks in the PP layer become visible, similar to the image taken of an affected backsheet with a scanning acoustic microscope in Figure 3c. This method can serve as a first indication of cracks inside the layer structure. A clear disadvantage of this method is that only the cell gaps can be analyzed and cracks along the busbars remain undetected.

In order to identify the polymer materials and locate chemical degradation features, further analysis of extracted backsheet samples such as FTIR is typically applied. A high resolution FTIR-ATR spectrum thereby 


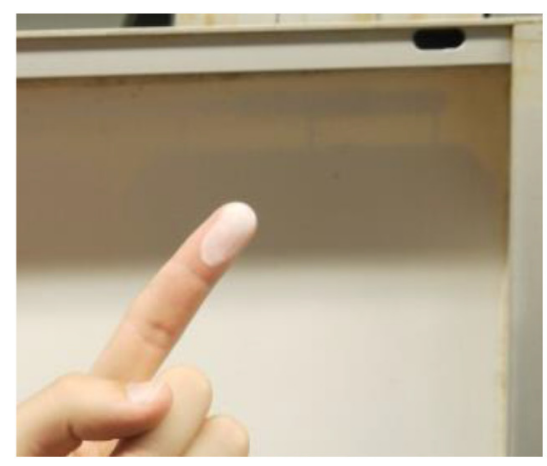

(a)

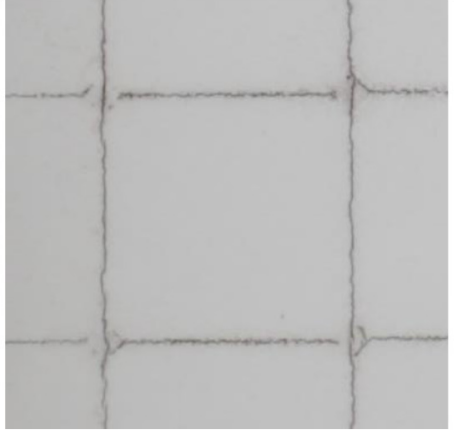

(b)

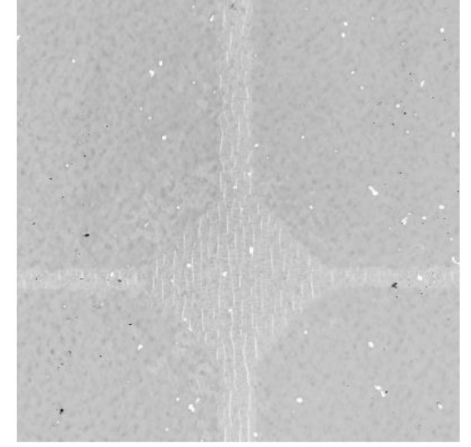

(c)

Fig. 3. Images from different degradation features; (a) Chalking on rear side of a polyamide backsheet [9]; (b) Cracked AAA backsheet; (c) Small cracks, indicating a damaged PP layer in a F-C/PET/PP backsheet.

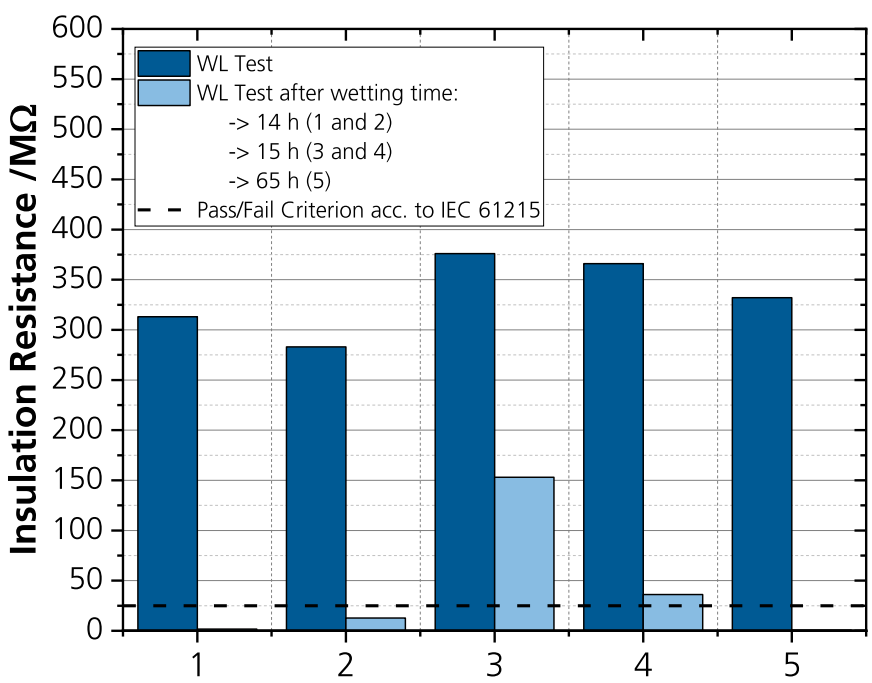

Fig. 4. Wet leakage current test results of five exemplary modules with F-C/PET/PP backsheets from two power plants that have been measured initially and after extended wetting times in a water bath of $14 \mathrm{~h}$ (modules 1 and 2), $15 \mathrm{~h}$ (modules 3 and 4 ) and $65 \mathrm{~h}$ (module 5).

requires the extraction and mechanical separation of individual backsheet layers. This bears different risks of misinterpretation of the results, e.g., when the adhesion forces do not allow clear separation of the layers, the exact layer structure is unknown or signals from the adhesion layers or other very thin layers are interfering in the polymer spectra. A solution to these drawbacks is the combination of microscopy and FTIR spectroscopy (Fig. 5), as it has been previously used by several authors to characterize polymer materials [13-15]. The measurement of a cross-section sample allows the exact selection of individual backsheet layers via adjustment of the respective apertures.

The FTIR microscopy has the advantage over ATR spectroscopy that it can be used to select a very small measurement area. There is no need to scrape off the outer layer to measure the core of the backsheets, since a cross-section is used for the measurement. To obtain a suitable signal to noise ratio for the used device, a layer thickness above $100 \mu \mathrm{m}$ is recommended.

The PET layer, which can be found in the core of the backsheet in the left image of Figure 5 , is shown in a comparison of the FTIR-ATR spectrum with the FTIRmicroscope spectrum. As indicated by the vertical markers, all significant peaks of the FTIR-ATR spectrum can also be observed in the FTIR spectrum of the PET layer.

The spectrum of the outer layer of the APA backsheet, despite the noisier signal is sufficient for a qualitative statement: The FTIR-microscope spectrum has five peaks that coincide well with the FTIR-ATR spectrum: Peaks at $1097 \mathrm{~cm}^{-1}, 1240 \mathrm{~cm}^{-1}, 1719 \mathrm{~cm}^{-1}, 2846 \mathrm{~cm}^{-1}$ and $2918 \mathrm{~cm}^{-1}$.

Thus, the results provide evidence that the FTIR microscope can be a suitable method for the determination of the backsheet polymers, depending on the polymer being examined. However, it should be noted that the signal can usually only be used between $3500-1000 \mathrm{~cm}^{-1}$, since a high noise level occurs in other measurement ranges. The investigated polymer should therefore have characteristic peaks in this area by means of which it can be identified. The polypropylene (PP) layer in the F-C/PET/PP backsheet also proved to be well suited for examination with the FTIR microscope. The technique has shown that generally, the spectrum yields a higher noise background for a thinner polymeric layer, such as the fluoropolymers in the PVDF.

As another analysis technique, scanning acoustic microscopy was used to uncover backsheet cracking in specific layers non-destructively [10]. The resolution of the measurement is however limited and might be not appropriate to investigate cracks in the $\mu \mathrm{m}$-range. The signal to noise ratio in greater depth could further be increased by the adaption of transducers with higher excitation frequencies. Figure 6 right (a and b) presents acoustic micrographs obtained from different time of flight (TOF) ranges inside the multilayered backsheets.

In order to validate the visualization of the location of these cracks and the depth profile of the PV module, acoustic line-scans along two different positions were executed, as presented in Figure 7 for a module with 


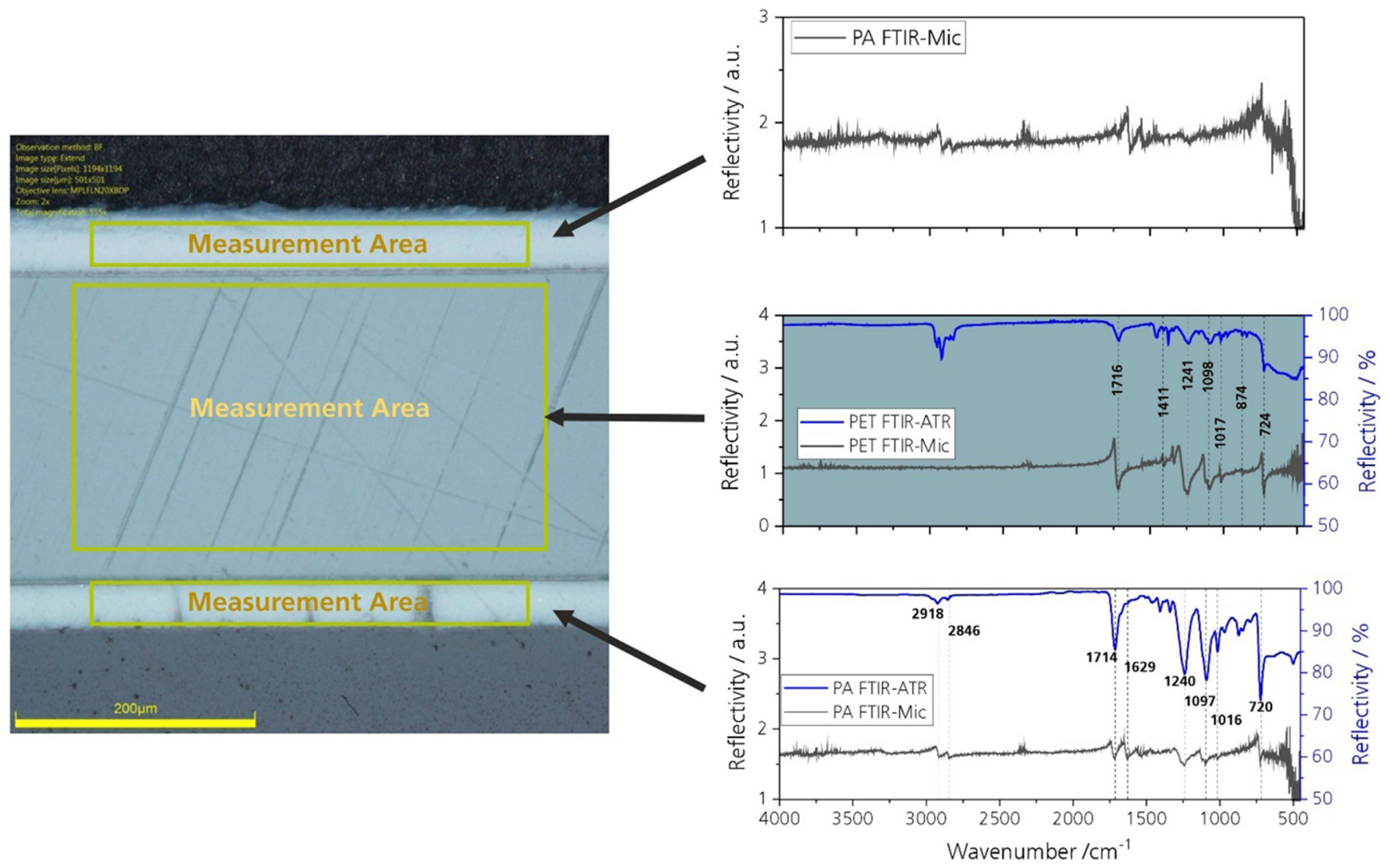

Fig. 5. Left: Microscopy image of the multilayer PA/PET/PA backsheet cross-section in a PV module. Right: Comparison of FTIR microscopy spectra (black) of cross-section samples and ATR-FTIR spectroscopy spectra (blue) of separated backsheet surface layers.
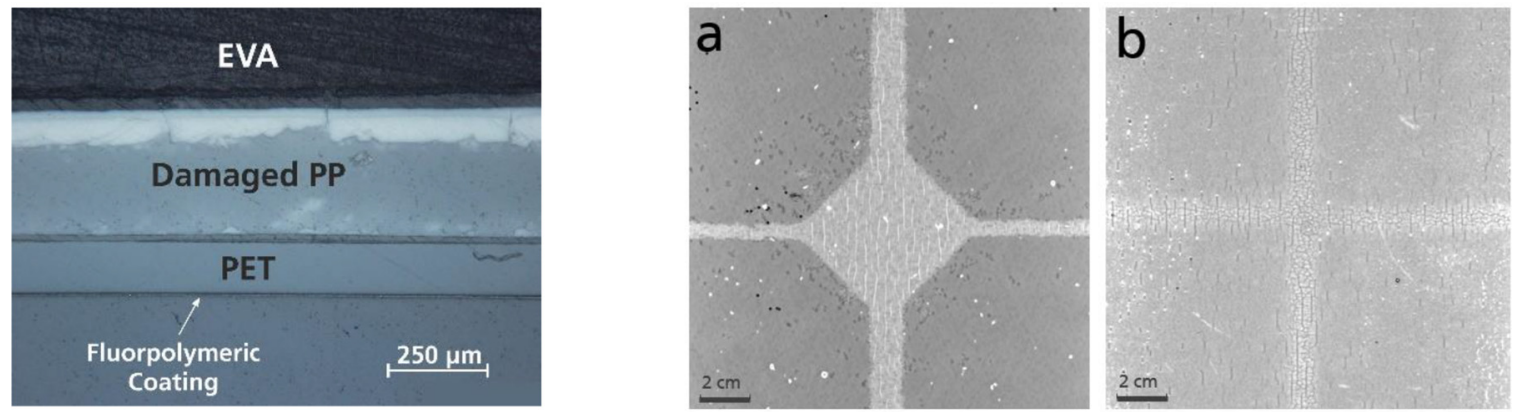

Fig. 6. Left: Cross section of a F-C/PET/PP coating with damaged PP layer. Right: Acoustic images of the multilayer backsheets in a field-exposed PV module; (a) core/inner layers interface of F-C/PET/PP backsheet; (b) outer/core layers interface of PA/PET/PA backsheet.

F-C/PET/PP backsheet and in Figure 8 for the module with APA backsheet. The corresponding backsheet cracks in the upper lines are marked with arrows for the comparison between the acoustic micrographs in the middle and the acoustic cross-section on the top.

The acoustic cross-sections confirm the same crack positions within the layer stack of the different modules. For modules with F-C/PET/PP backsheet, the cracks are visible at the 4 th interface starting from the top line, which means at the backsheet inner/EVA layer interface. This coincides well with the results of optical microscopy (destructive) of the same material (Fig. 6, left). For the module with APA backsheet, the cracks appear slightly earlier at the $2 n d$ interface, i.e., the outer $\mathrm{PA} /$ core PET layer interface. This coincides well with the results of optical microscopy (destructive) of the same material (Fig. 5, left), whereas, for the module with APA backsheet, the cracks appear slightly earlier at the 2nd interface, i.e., the outer $\mathrm{PA} /$ core $\mathrm{PET}$ layers interface. 


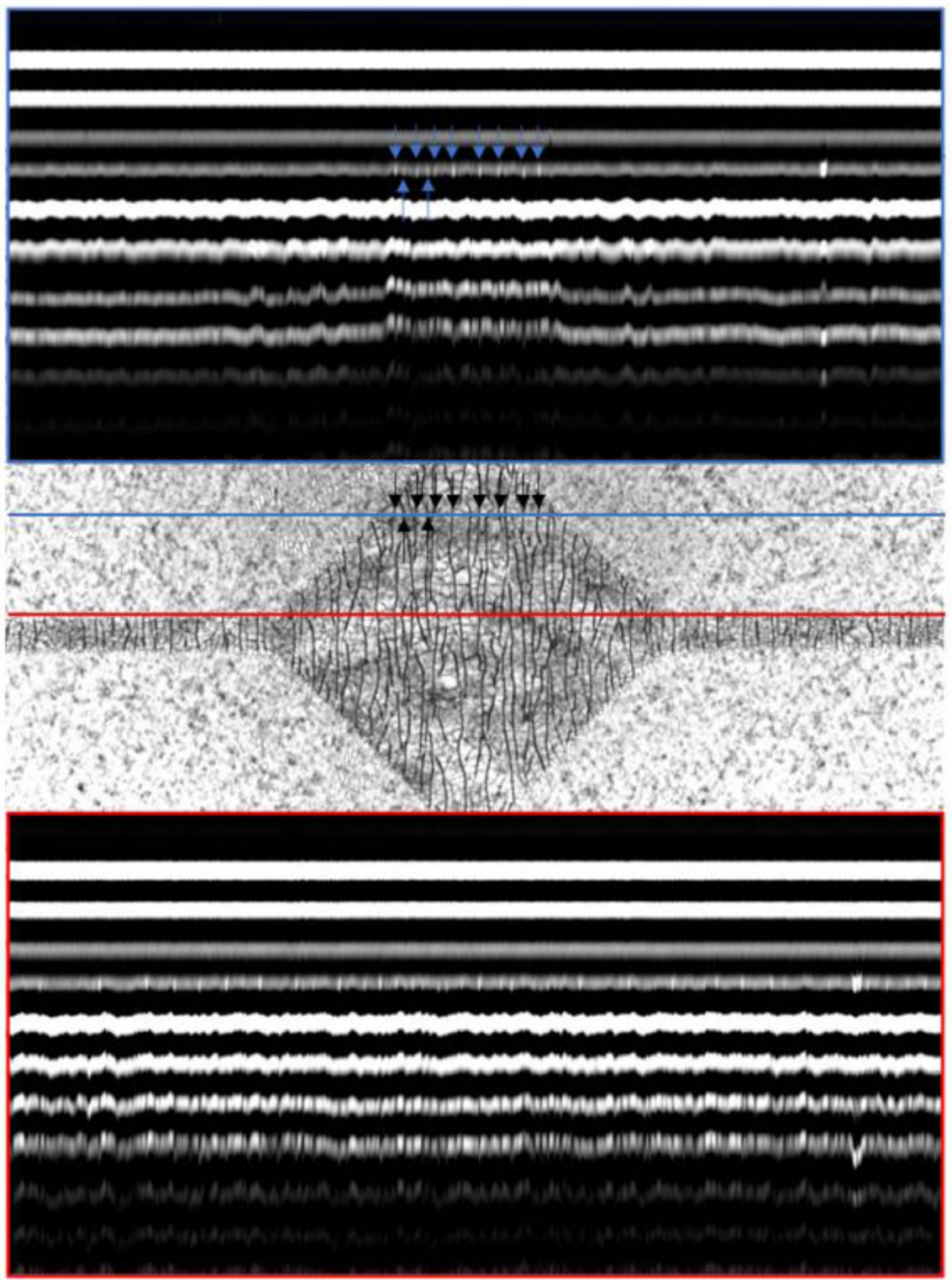

Fig. 7. Acoustic cross-sections of a module with F-C/PET/PP backsheet revealing internal cracks (top and bottom), taken at the lines indicated in the micrograph (middle).

\section{Conclusion}

In this work, we present analysis results of 279 modules from 26 power plants which have been investigated and characterized in our lab. A variety of different failures in a number of modules with different backsheet types could be identified. Besides backsheet chalking, crack formation in different material layers of the backsheets was one of the most prominent degradation features. Our observations in polyamide layer structures suggest a high risk of backsheet cracking when backsheet chalking is observed. Crack formation is critical, since it can lead to reduced electrical insulation properties and consequently increased electrical hazards. In this context, it has been shown, that only one sixth of all modules did not pass the WL insulation requirements according to IEC 61215-2:2021. Another five modules showed significantly reduced insulation resistances only after an increased wetting time, which might be an indication that a drying-out process could have happened during storage and /or transportation. This indicates that a single WL test applied to modules from field that are measured in a lab is not sufficient to state the insulation stability.

Backsheets with two specific material combinations were found to be most frequently defective: AAA and $\mathrm{F}-\mathrm{C} / \mathrm{PET} / \mathrm{PP}$. However, the data also shows that the issue generally applies to a wide range of materials and manufacturers and cannot be narrowed down to 


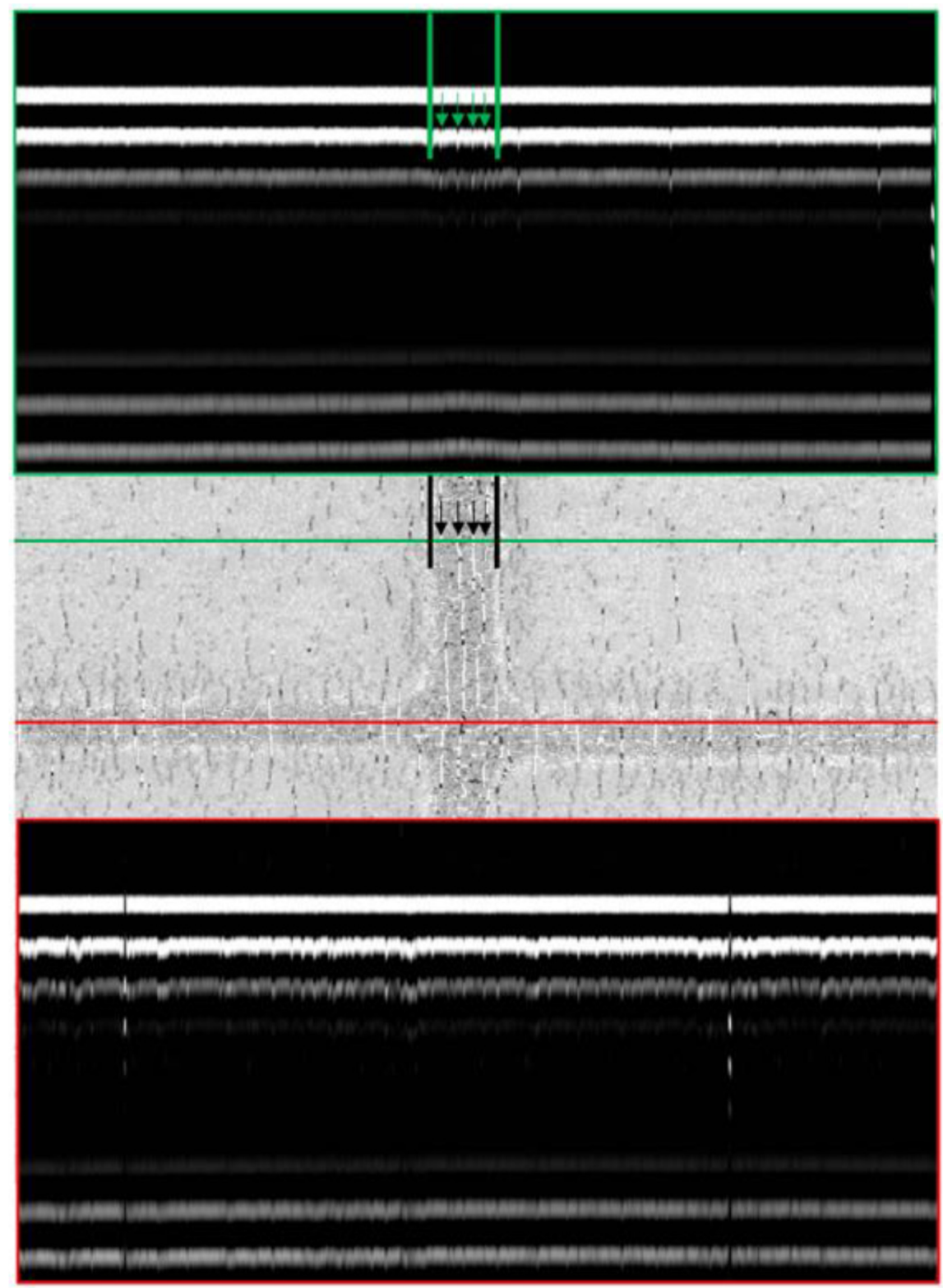

Fig. 8. Acoustic cross-sections of a module with PA/PET/PA backsheet with polyamide outer layer cracking (top and bottom), taken at the lines indicated in the micrograph (middle).

individual products. Furthermore, it has to be considered that backsheet failures typically develop over the course of several years before detection. Due to the dynamic market, our findings do not directly correspond to the products available on the market today. Due to the widespread use of backsheets in the past, failures of modules from further power plants have to be expected in the future. Additionally, new critical backsheet materials and products could become more relevant, as also reported by others [13]. Understanding and early detection of the failures is hence essential to develop durable bill of materials that ensure a high module quality in the future.
Suitable characterization methods were introduced as straightforward techniques for investigating such failures. Firstly, the "flashlight test" was found to be a fast and lowtech method to identify backsheet cracks in cell gaps. Secondly, FTIR microscopy allows the precise identification of the chemical structure of the different backsheet layers at the cross-sectional level, which avoids possible error sources of other methods.

Furthermore, the application of SAM as a nondestructive method for the visualization of the backsheet cracking was presented. The method provides a nondestructive way to evaluate the condition of the backsheet not only at the outer surface but also in bulk layers and could 
thereby uncover internal backsheet cracking which was invisible from the outside. Additionally, the acoustic crosssections allowed the depth profile analysis of the multilayer backsheets, and the exact determination of cracks depths.

The combination of all presented methods poses new possibilities of approaching degradation features found in field-aged PV modules. The presented methods can serve as meaningful tools to estimate the condition of modules when the value of a power plant needs to be determined or the plant reaches the end of its expected lifetime (e.g., runout of feed-in tariffs). In these cases, operators or investors may need more detailed information about the module condition, regardless of whether the modules show clear visual or measurable failure patterns.

\section{Author contribution statement}

J. Markert and P. Gebhardt supervised the measurements of the field-aged PV modules in the lab and performed the result evaluation together with D. Philipp. D. E. Mansour and S. Kotterer provided the experimental data, as well as the evaluation of the analytical results acquired with the different methods. The manuscript was prepared by J. Markert, D. E. Mansour, S. Kotterer and P. Gebhardt. All authors were involved in discussions on the experimental data and D. Philipp and P. Gebhardt provided feedback and corrections throughout the preparation process of the work.

The authors acknowledge the funding from the German Federal Ministry for Economic Affairs and Energy (FKz.: 0324354 "proModul").

\section{References}

1. Y. Lyu, A. Fairbrother, M. Gong, J.H. Kim, X. Gu, M. Kempe, S. Julien, K.-T. Wan, S. Napoli, A. Hauser et al., Solar Energy 199, $425(2020)$
2. G.C. Eder, Y. Voronko, G. Oreski, W. Mühleisen, M. Knausz, A. Omazic, A. Rainer, C. Hirschl, H. Sonnleitner, Solar Energy Materials and Solar Cells 203, 110194 (2019)

3. P. Lechner, H. Wirth, J. Schnepf, S. Hummel, D. Geyer, B. Weinreich, R. Haas, in Proceedings of the 36th European Photovoltaic Solar Energy Conference and Exhibition, 2019, pp. 930-934

4. M. Köntges, G. Oreski, U. Jahn, M. Herz, P. Hacke, K.-A. Weiss, G. Razongles, M. Paggi, D. Parlevliet, T. Tanahashi et al., Assessment of Photovoltaic Module Failures in the Field. Report IEA-PVPS T13-09:2017, 2017

5. D.E. Mansour, C. Barretta, L. Pitta Bauermann, G. Oreski, A. Schueler, D. Philipp, P. Gebhardt, Sustainability 12, 5208 (2020)

6. G.C. Eder, Y. Lin, Y. Voronko, L. Spoljaric-Lukacic, Energies 13, 1903 (2020)

7. J.E. Mark, Physical properties of polymers handbook. Acoustic Properties of Polymers (Springer, New York, 2006)

8. A.P. Patel, A. Sinha, G. Tamizhmani, IEEE J. Photovolt. 10, $607(2020)$

9. P. Gebhardt, L. Pitta Bauermann, D. Philipp, in Proceedings of the 35th European Photovoltaic Solar Energy Conference and Exhibition (EU PVSEC); Brussels, Belgium, 2018, pp. 1097-1100

10. D.E. Mansour, S. Kotterer, D. Philipp, P. Gebhardt (Eds.) 37th European Photovoltaic Solar Energy Conference and Exhibition, 2020

11. C.-C. Lin, Y. Lyu, D.S. Jacobs, J.H. Kim, K.-T. Wan, D.L. Hunston, X. Gu, Prog. Photovolt: Res. Appl. 89, 139 (2018)

12. International Electrotechnical Commission, IEC 61215: Terrestrial photovoltaic (PV) modules. Design qualification and type approval - Part 2: Test Procedures International standard Norme internationale, IEC 61215-2:2021 (International Electrotechnical Commission, Geneva, Switzerland, 2021)

13. J. Tracy, K.R. Choudhury, W. Gambogi, T. Felder, L. Garreau-Iles, H. Hu, T.J. Trout, R. Khatri, X. Ji, Y. Heta in Proceedings of the 46th Photovoltaic Specialists Conference (IEEE PVSC), IEEE, 2019, pp. 874-879

14. Y. Voronko, B.S. Chernev, G.C. Eder, Appl. Spectrosc. 68, $584(2014)$

15. G. van Dalen, P.C.M. Heussen, R. den Adel, R.B.J. Hoeve, Appl. Spectrosc. 61, 593 (2007)

Cite this article as: Jochen Markert, Sandra Kotterer, Djamel Eddine Mansour, Daniel Philipp, Paul Gebhardt, Advanced analysis of backsheet failures from 26 power plants, EPJ Photovoltaics 12, 7 (2021) 